\title{
On solving variational inequalities defined on fixed point sets of multivalued mappings in Banach spaces
}

\author{
Hong-Kun Xu and Luigi Muglia
}

\begin{abstract}
We are concerned with the problem of solving variational inequalities which are defined on the set of fixed points of a multivalued nonexpansive mapping in a reflexive Banach space. Both implicit and explicit approaches are studied. Strong convergence of the implicit method is proved if the space satisfies Opial's condition and has a duality map weakly continuous at zero, and the strong convergence of the explicit method is proved if the space has a weakly continuous duality map. An essential assumption on the multivalued nonexpansive mapping is that the mapping be single valued on its nonempty set of fixed points.
\end{abstract}

Mathematics Subject Classification. 47J20, 47J25, 49J40.

Keywords. Accretive operator, variational inequality, iterative method, multivalued nonexpansive mapping, opial condition, duality map.

\section{Introduction}

In this paper, we are concerned with the problem of solving variational inequalities in Banach spaces. More precisely, let $X$ be a Banach space, let $C$ be a nonempty closed convex subset of $X$, and let $A: \subset D(A) \subset X \rightarrow X$ be an operator, such that $C \subset D(A)$. The variational inequality (VI), associated with $A$ and $C$, is the problem of finding a point $x^{*} \in C$ with the property:

$$
\left\langle A x^{*}, j\left(x-x^{*}\right)\right\rangle \geq 0 \text { for all } x \in C,
$$

where $j\left(x-x^{*}\right) \in J\left(x-x^{*}\right)$ and $J$ is the normalized duality map on $X$ defined by:

$$
J(x):=\left\{x^{*} \in X^{*}:\left\langle x, x^{*}\right\rangle=\|x\|^{2},\left\|x^{*}\right\|=\|x\|\right\}, \quad x \in X .
$$

It is easily understood that the existence and uniqueness of solutions of VI (1.1) require the operator $A$ to satisfy certain conditions. For instance, if $A$ is $\eta$-strongly accretive for some $\eta>0$ (i.e., $\langle A x-A y, j(x-y)\rangle \geq \eta\|x-y\|^{2}$ 
for all $x, y \in C)$, then VI (1.1) has at most one solution. Indeed, if $w$ and $z$ are solutions of VI (1.1), then adding up the inequalities

$$
\langle A w, j(z-w)\rangle \geq 0 \quad \text { and } \quad\langle A z, j(w-z)\rangle \geq 0
$$

yields $-\eta\|z-w\|^{2} \geq-\langle A z-A w, j(z-w)\rangle \geq 0$; hence, $z=w$.

Observe that in a Hilbert space $H$, we may rewrite VI (1.1) as a variational inequality that is defined on the fixed point set $F i x(T)$ of a nonexpansive mapping $T: C \rightarrow C$ (i.e., $\|T x-T y\| \leq\|x-y\|$ for $x, y \in C$ ):

$$
\left\langle A x^{*}, x-x^{*}\right\rangle \geq 0 \quad x \in \operatorname{Fix}(T) .
$$

As a matter of fact, we may take $T=P_{C}$ to be the metric projection onto $C$. This shows an equivalence of VIs defined on an arbitrary closed convex subset $C$ and VIs defined on the fixed point set of an arbitrary nonexpansive mapping on $C$.

This is, however, no longer true in the setting of Banach spaces, due to the fact that there exist closed convex sets of a Banach space $X$ which are not fixed point sets of nonexpansive mappings $T: X \rightarrow X$. Such an example can be found in [1, p. 25]. However, if $X$ is strictly convex, then every nonexpansive mapping $T: C \rightarrow C$ has a closed convex fixed point set $\operatorname{Fix}(T)$.

In this paper, we will study VI (1.3) in the case where the feasible set $C$ is the set of fixed points of a multivalued nonexpansive mapping in a Banach space. In what follows, we will denote by $C B(X)$ the collection of nonempty closed bounded subsets of $X$ and by $K(X)$ the collection of nonempty compact subsets of $X$, respectively. Moreover, we will use $H(\cdot, \cdot)$ to stand for the Hausdorff metric on $C B(X)$ (see definition in Section 2).

Now, recall that a multivalued mapping $T: C \rightarrow C B(C)$ is said to be an $\eta$-contraction if there exists $\eta \in[0,1)$, such that:

$$
H(T x, T y) \leq \eta\|x-y\|, \quad x, y \in C .
$$

In the case of $\eta=1$, that is:

$$
H(T x, T y) \leq\|x-y\|, \quad x, y \in C,
$$

$T$ is said to be a (multivalued) nonexpansive mapping. Recall that a point $x \in C$ is said to be a fixed point of a multivalued mapping $T: C \rightarrow C B(C)$ if $x \in T x$. The set of fixed points of $T$ is denoted as Fix $(T)$. That is, Fix $(T)=$ $\{x \in C: x \in T x\}$.

Existence of fixed points of multivalued mappings is, in general, intricate and, sometimes, surprising. For instance, Nadler [2] proved the existence of a fixed point of a multivalued contraction on a complete metric space, but not uniqueness, as opposed to the single-valued contraction which has a unique fixed point.

Fundamental results about the existence of fixed points for multivalued nonexpansive mappings can be found in Lami Dozo [3] in the setting of Banach spaces satisfying the Opial condition and in Lim [4] in the setting of uniformly convex Banach spaces. Let us remark that the set of fixed points of a multivalued nonexpansive mapping $T: C \rightarrow K(C)$ on a strictly convex Banach spaces is, in general, not a convex set (see [5, Section 3]). More 
existence results on fixed points of multivalued mappings may be found in the survey article [6]. In this connection, see also the paper by Reich [7].

Observe that approximation methods for fixed points of multivalued mappings seem to be much less developed than those for single-valued mappings. As a matter of fact, some well-known method for approximating fixed points of single-valued nonexpansive mappings may fail to work for multivalued nonexpansive mappings. For instance, a counterexample, due to Pietramala [8], shows that the famous Browder's approximation result [9, Theorem 1] for a single-valued nonexpansive mapping cannot be extended to the genuine multivalued case even in the Euclidean space $\mathbb{R}^{2}$.

On the other hand, Lopez and $\mathrm{Xu}[10]$ verified the convergence of Browder's approximation for multivalued nonexpansive-type mappings upon supposing that $F i x(T)=\{z\}$ is singleton. Sahu [11] and later Jung [12] extended Lopez-Xu's result to the setting of a uniformly convex Banach space and the setting of reflexive spaces with a uniformly Gateaux differentiable norm, respectively. Moreover, they relaxed the condition of [10] by supposing that $T$ is single valued on the set of fixed points, i.e., $T w=\{w\}$ if $w \in F i x(T)$.

Nevertheless, Jung [12] declares that, in his opinion, this last hypothesis seems to be restrictive; therefore, he asked the question if it could be dropped. In 2007, Shahzad and Zegeye [13] gave a partial answer to Jung's question: noting that, in view of the example shown by Pietramala, it was not completely possible to omit it. They proposed an alternative approach by introducing in their iterative sequence the metric projection $P_{T}$ as follows:

$$
P_{T}(x):=\{u \in T x:\|x-u\|=d(x, T x)\} .
$$

where $T: C \rightarrow K(C)$ is given and $d(x, T x):=\inf _{y \in T x}\|x-y\|$ is the distance to $T x$ from $x$. Instead of studying the asymptotic behavior of the implicitly defined curve $x_{t}$ :

$$
x_{t}=t T\left(x_{t}\right)+(1-t) u, \quad \text { as } t \rightarrow 1 ;
$$

Shahzad and Zegeye [13] studied the asymptotic behavior of the implicitly defined net $\left(x_{t}\right)$ :

$$
x_{t}=t P_{T}\left(x_{t}\right)+(1-t) u, \quad \text { as } t \rightarrow 1 .
$$

The following result was proved in [14] (also in [15]).

Theorem 1.1. Let $X$ be a uniformly convex Banach space with a uniformly Gateaux differentiable norm, $C$ be a nonempty closed convex subset of $X$, and $T: C \rightarrow K(E)$ be such that $P_{T}$ is nonexpansive. Suppose that $C$ is a nonexpansive retract of $X$, and that for each $t \in(0,1)$, the contraction $S_{t}$ defined by:

$$
S_{t} x=t P_{T} x+(1-t) f(x)
$$

has a fixed point $x_{t} \in C$, where $f: C \rightarrow C$ is a contraction with constant $\beta \in[0,1)$. Then, $T$ has fixed points if and only if $\left(x_{t}\right)$ remains bounded as $t \rightarrow 1^{-}$; in this case, $\left(x_{t}\right)$ strongly converges, as $t \rightarrow 1^{-}$, to a fixed point of $T$. 
Remark 1.2. In Theorem 1.1, the authors supposed that $C$ is a nonexpansive retract of the space $X$. The existence of such a nonexpansive retract for $C$ remains an unsolved problem in general (the answer is, however, affirmative [16] if $C$ is the set of fixed points of a nonexpansive mapping in a uniformly smooth Banach space). Recall that the classical metric projection onto a closed convex $C$ in Banach spaces is a continuous mapping but not necessarily nonexpansive (though true in Hilbert spaces). Moreover, it is proved in [1] that if $X$ is not a Hilbert space with $\operatorname{dim} X \geq 3$, then not every closed convex subset of $X$ is a nonexpansive retract of the space. In this connection, see also Reich [17] and Kopecká-Reich [18].

Note that for $f(x)=u$, the main theorem in [13] can be recovered from Theorem 1.1.

Iteration methods based on (1.4), which use contractions as a regularizer of nonexpansive mappings, are known as viscosity approximations, which amount to selecting a particular fixed point of a given nonexpansive mapping. This notion was first introduced to optimization by Attouch [19] and then extended to nonexpansive mappings by Moudafi [20] in the Hilbert space setting (see [21] for an extension to the setting of uniformly smooth Banach spaces). A novel element of this kind of iteration methods is the strong convergence to the unique solution of the variational inequality (VI):

$$
\left\langle(I-f) x^{*}, j\left(y-x^{*}\right)\right\rangle \geq 0 \text { for all } y \in F i x(T),
$$

where $(I-f)$ is a strongly monotone operator. In the particular case $f(x)=u$ and $X=H$, VI (1.5) becomes:

$$
\left\langle x^{*}-u, y-x^{*}\right\rangle \geq 0 \text { for all } y \in F i x(T) .
$$

This is equivalent to the minimum problem $\min _{x \in F i x(T)}\|x-u\|^{2}$.

The multivalued case of viscosity approximations has been studied by Cui et al. [22] in the setting of a reflexive space with a weakly sequentially continuous normalized duality mapping. Let us note that, in their paper, the authors assumed that $T$ is single valued on Fix $(T)$. Wu and Zhao [23] studied a viscosity method in the setting of uniformly convex and smooth Banach spaces; Panyanak and Suantai [24] considered the viscosity approximation method in geodesic spaces.

In 2017, Xu et al. [25] published a note to give the convergence analysis of the implicit Mann iteration process:

$$
x_{n+1}=\left(1-\tau_{n}\right) x_{n}+\tau_{n} y_{n+1},
$$

where $y_{n+1} \in T x_{n+1}$ and, in particular, weak convergence is proved in a uniformly convex Banach space that satisfies Opial's property.

In the paper [25], besides the main interesting results, we find other interesting elements:

- the authors showed examples of implicit and explicit Mann iterations that fail to converge if the condition $T w=\{w\}$ for $w \in F i x(T)$ is dropped. 
- The authors pointed out two gaps in the proof of the main result in [26].

Let us spend a few words about one of the gaps, because it connects to a very useful tool.

In many papers on iterative methods (see, e.g., $[12,13,15,26])$, Banach limits (see $[27$, p. 26]) are used to define a function $\phi$ by:

$$
\phi(x):=\operatorname{LIM}_{n \rightarrow \infty}\left\|x_{n}-x\right\|^{2}, \quad x \in X,
$$

where $\left(x_{n}\right)$ is a bounded sequence in $X$ (which is generated by an iterative method). It is easily found that $\phi$ is continuous, convex, and coercive (i.e., $\phi(x) \rightarrow \infty$ as $\|x\| \rightarrow \infty)$. Then, the reflexivity of the space $X$ ensures that $\phi$ attains its minimum on a closed convex set $C$. Let $p \in C$ be a minimizer of $\phi$ over $C$. If $C$ is a nonexpansive retract of $X$, this minimum is a global minimum on $X$. The key is to prove that $p$ is a fixed point of $T$. Using compactness arguments, they proved that, given $\left(w_{n}\right)_{n \in \mathbb{N}} \subset T p$, there exists a subsequence strongly convergent to $w \in T p$ (wrongfully indicated by the same sequence $\left.\left(w_{n}\right)_{n \in \mathbb{N}}\right)$. Therefore, using the formula that defines the iteration, they proved that:

$$
\phi(w)=\operatorname{LIM}_{n \rightarrow \infty}\left\|x_{n}-w\right\|^{2} \leq \ldots \leq \operatorname{LIM}_{n \rightarrow \infty}\left\|x_{n}-p\right\|^{2}=\phi(p)=\min _{X} \phi,
$$

and then drew the conclusion that $w=p$ and thus $p \in T p$.

Unfortunately, the above argument holds for a subsequence $\left(w_{n_{k}}\right)_{k \in \mathbb{N}}$ of $\left(w_{n}\right)_{n \in \mathbb{N}}$ only, and so (1.7) holds for a subsequence $\left(x_{n_{k}}\right)$ only; that is, the correct statement of (1.7) should be:

$$
\operatorname{LIM}_{k \rightarrow \infty}\left\|x_{n_{k}}-w\right\|^{2} \leq \ldots \leq \operatorname{LIM}_{k \rightarrow \infty}\left\|x_{n_{k}}-p\right\|^{2} .
$$

Consequently, the conclusion $w=p$ cannot be drawn from (1.8). Notice that Banach limits are sensitive to subsequences, as the following simple example shows: consider the real sequence $a_{n}=1+(-1)^{n}$; then, we have:

$$
\operatorname{LIM}_{n \rightarrow \infty} a_{n}=1, \operatorname{LIM}_{n \rightarrow \infty} a_{2 n+1}=0, \operatorname{LIM}_{n \rightarrow \infty} a_{2 n}=2 .
$$

Therefore, the claim $w=p$ in the above proof is not convinced.

In this paper, taking into account the above background, we study the VI (1.3) in the case where the feasible set $C$ is the set of fixed points of a multivalued nonexpansive mapping and the controlling operator $A$ is strongly accretive, which will be relabeled as $D$. Namely, our VI is restated as finding a point $x^{*} \in \operatorname{Fix}(T)$ satisfying the property:

$$
\left\langle D x^{*}, j\left(x-x^{*}\right)\right\rangle \geq 0 \text { for all } x \in F i x(T),
$$

where $T$ is a multivalued nonexpansive mapping on a Banach space $X$ having a nonempty set of fixed points. The VI (1.9) encompasses, as a particular case, viscosity problems (1.5) if $D=I-f$ and minimum problems on Hilbert spaces (1.6) if $D=I-u$.

We will introduce implicit and explicit iterative approaches that generate a sequence converging strongly to a solution of VI (1.9). In our argument, we do not use Banach limit. 
The rest of the paper is organized as follows. Section 2 includes definitions of multivalued nonexpansive mappings and some Banach space concepts. We also present some basic tools which are used in the proof of the main results of the paper.

In Section 3, we state and prove the main results of this paper on implicit and explicit methods for solving a variational inequality which is defined on the set of fixed points of a multivalued nonexpansive mapping in a reflexive Banach space that satisfies Opial's condition and has a duality map $J_{\varphi}$ weakly continuous at zero. In addition, we will raise some open problems.

\section{Preliminaries}

Let $(X,\|\cdot\|)$ be a Banach space and let $C$ be a nonempty closed convex subset of $X$. Denote by $C B(C)$ the collection of all nonempty closed bounded subsets of $C$ and by $K(C)$ the collection of nonempty compact subsets of $C$, respectively.

Recall that the Hausdorff distance between $A, B \in C B(C)$ is defined as:

$$
H(A, B):=\max \left\{\sup _{a \in A} d(a, B), \sup _{b \in B} d(b, A)\right\},
$$

where $d(x, E):=\inf _{e \in E}\|e-x\|$ for $x \in X$ and subset $E \subset X$.

The lemma below will be used to verify that our approaches are well defined; here, we include the proof for the sake of completeness.

Lemma 2.1. Let $A, B \subset X$ and $x, y \in X$. For any given $\lambda \in(0,1)$, let $A_{\lambda}:=$ $\lambda x+(1-\lambda) A$ and $B_{\lambda}:=\lambda y+(1-\lambda) B$. Then:

$$
H\left(A_{\lambda}, B_{\lambda}\right) \leq \lambda\|x-y\|+(1-\lambda) H(A, B) .
$$

Proof. Let $a_{\lambda}:=\lambda x+(1-\lambda) a$ and $b_{\lambda}:=\lambda y+(1-\lambda) b$ with $a \in A$ and $b \in B$, respectively. Then:

$$
\begin{aligned}
d\left(a_{\lambda}, B_{\lambda}\right) & =\inf _{b_{\lambda} \in B_{\lambda}}\left\{\left\|a_{\lambda}-b_{\lambda}\right\|\right\} \\
& \leq \inf _{b \in B}\{\lambda\|x-y\|+(1-\lambda)\|a-b\|\} \\
& =\lambda\|x-y\|+(1-\lambda) d(a, B) .
\end{aligned}
$$

In a similar way, $d\left(b_{\lambda}, A_{\lambda}\right) \leq \lambda\|x-y\|+(1-\lambda) d(b, A)$; therefore:

$$
\begin{aligned}
H\left(A_{\lambda}, B_{\lambda}\right) & =\max \left\{\sup _{a_{\lambda} \in A_{\lambda}} d\left(a_{\lambda}, B_{\lambda}\right), \sup _{b_{\lambda} \in B_{\lambda}} d\left(A_{\lambda}, b_{\lambda}\right)\right\} \\
& \leq \lambda \| x-y \mid+(1-\lambda) \max \left\{\sup _{a \in A} d(a, B), \sup _{b \in B} d(A, b)\right\} \\
& =\lambda\|x-y\|+(1-\lambda) H(A, B) .
\end{aligned}
$$


Recall that the norm $\|\cdot\|$ of a Banach space $X$ is said to be Gateaux differentiable (and $X$ is said to be smooth) if the limit

$$
\lim _{t \rightarrow 0} \frac{\|x+t y\|-\|x\|}{t}
$$

exists for all $x, y$ in the unit sphere of $X$.

It is known (cf. [27]) that $X$ is smooth if and only if the normalized duality map $J_{X}$ defined by (1.2) is single valued. Another notion is the Opial's condition [28]: a Banach space $X$ is said to satisfy Opial's condition if

$$
\liminf _{n \rightarrow \infty}\left\|w_{n}-w\right\|<\liminf _{n \rightarrow \infty}\left\|w_{n}-p\right\|
$$

whenever $\left(w_{n}\right)$ is a sequence in $X$ weakly convergent to $w$ and $p \neq w$. Opial's condition plays an important role in the fixed point theory (see [3,29-31]).

The notion of the normalized duality map can be extended to more general case. Recall that a function $\varphi: \mathbb{R}^{+} \rightarrow \mathbb{R}^{+}$is said to be a gauge if it satisfies the properties: (i) $\varphi(0)=0$, (ii) $\varphi$ is continuous and strictly increasing, and (iii) $\varphi(t) \rightarrow+\infty$ as $t \rightarrow+\infty$. Associated to a gauge $\varphi$ is the duality map $J_{\varphi}$ defined by [32]:

$$
J_{\varphi}(x)=\left\{x^{*} \in X^{*}:\left\langle x, x^{*}\right\rangle=\|x\|\left\|x^{*}\right\|, \varphi(\|x\|)=\left\|x^{*}\right\|\right\} .
$$

When $\varphi(t)=t^{p-1}$ for some $p \in(1,+\infty)$, the duality map is referred to as the generalized duality map of order $p$. In particular, when $p=2$, we recover the normalized duality map $J$. It is a well-known result due to Asplund (see, for instance, $[33,34])$ that $J_{\varphi}(x)$ is the subdifferential $\partial \Phi(\|x\|)$ of the convex functional $\Phi(t)$ defined by $\Phi(t)=\int_{0}^{t} \varphi(s) d s$. The relationship between $J_{\varphi}$ and $J$ is given by the equation:

$$
J(x) \varphi(\|x\|)=\|x\|^{2} J_{\varphi}(x), \quad x \in X .
$$

Following Browder [32], we say that a Banach space $X$ has a weakly sequentially continuous duality map $J_{\varphi}$ for some gauge $\varphi$ if $J_{\varphi} x_{n} \rightarrow J_{\varphi} x$ in the weak* topology of $X^{*}$ whenever $x_{n} \rightarrow x$ in the weak topology of $X$. The following result is useful in fixed point theory and geometry of Banach spaces $[35,36]$.

Lemma 2.2. Let $X$ be a Banach space with a weakly sequentially continuous duality map $J_{\varphi}$ for some gauge $\varphi$. Assume that $\left(x_{n}\right)$ is a sequence in $X$ weakly converging to $x^{*}$. Then:

$$
\limsup _{n \rightarrow \infty} \Phi\left(\left\|x_{n}-x\right\|\right)=\limsup _{n \rightarrow \infty} \Phi\left(\left\|x_{n}-x^{*}\right\|\right)+\Phi\left(\left\|x-x^{*}\right\|\right)
$$

for all $x \in X$. In particular, $X$ satisfies Opial's condition (but not vice versa $[31,38])$.

Lemma 2.3. [31, Theorem 2] Let $X$ be a Banach space with the properties:

(i) $X$ satisfies the weak Opial condition: $\liminf _{n \rightarrow \infty}\left\|x_{n}-x\right\| \leq \liminf _{n \rightarrow \infty}$ $\left\|x_{n}-y\right\|$ whenever $x_{n} \rightarrow x$ and $y \in X$.

(ii) The norm of $X$ is uniformly Gateaux differentiable. 
Then, the duality map $J_{\phi}$ associated with any gauge $\phi$ is weakly sequentially continuous at zero (but not necessarily weakly sequentially continuous at other nonzero points [38]).

Definition 2.4. (cf. [37]) Let $X$ be a Banach space and let $D: X \rightarrow X$ be an operator.

(i) We say that $D$ is $\eta$-strongly accretive for some $\eta \in(0,1)$ if, for every $x, y \in X$, there exists $j(x-y) \in J(x-y)$, such that:

$$
\langle D x-D y, j(x-y)\rangle \geq \eta\|x-y\|^{2} .
$$

(ii) We say that $D$ is $\tau$-strict pseudocontractive for some $\tau \in(0,1)$ if, for every $x, y \in X$, there exists $j(x-y) \in J(x-y)$, such that:

$$
\langle D x-D y, j(x-y)\rangle \leq\|x-y\|^{2}-\tau\|(I-D) x-(I-D) y\|^{2} .
$$

The next proposition, proved in [37], will be used in what follows to define our iterative approach.

Proposition 2.5. [37] Let $X$ be a smooth Banach space and let $D: X \rightarrow X$ be an operator. Given $\eta, \tau \in(0,1)$.

(i) If $D$ is $\tau$-strictly pseudocontractive, then $D$ is L-Lipschitzian with $L=$ $1+\tau^{-1}$.

(ii) If $D$ is $\eta$-strongly accretive and $\tau$-strictly pseudocontractive with $\eta+\tau>$ 1 , then $(I-\lambda D)$ is a $(1-\lambda \rho)$-contraction for all $\lambda \in(0,1)$, where $\rho=(1-\sqrt{(1-\eta) / \tau})$.

Remark 2.6. The statement (i) of Proposition 2.5 is not vice versa; for instance, the mapping $D x:=\frac{3}{2} x$ in the one-dim case is not a strict pseudocontractive mapping.

If $X=H$ is a Hilbert space, it is well known (see [38]) that if $D$ is an $\eta$-strongly monotone and $L$-Lipschitzian operator, then $(I-\lambda D)$ is a contraction if $0<\lambda<\frac{2 \eta}{L^{2}}$.

\section{Convergence results}

We begin with a simple example which shows that iterative methods for multivalued mappings are quite uncertain in terms of convergence.

Example 3.1. Define $T: \mathbb{R} \rightarrow 2^{\mathbb{R}}$ by $T x=\{x, x+1\}$ and let $f$ be a $\rho$ contraction for some $\rho \in[0,1)$. It is easily seen that $T$ is nonexpansive. Notice that every point $x$ is a fixed point of $T$; however, $T$ is not single valued on Fix $(T)$. Let us consider the implicit iteration:

$$
x_{n}=\alpha_{n} f\left(x_{n}\right)+\left(1-\alpha_{n}\right) x_{n}^{T},
$$

where $\alpha_{n} \in(0,1)$ and $x_{n}^{T} \in T x_{n}$. Notice that we have two options for $x_{n}^{T}$, namely, either $x_{n}^{T}=x_{n}$ or $x_{n}^{T}=x_{n}+1$. Consider the particular case where $f(x) \equiv u$ is constant. In addition, consider the choice $x_{n}^{T}=x_{n}$ for odd $n$ and $x_{n}^{T}=x_{n}+1$ for even $n$. It turns out that $x_{n}=u$ for odd $n$ and $x_{n}=u-1+\alpha_{n}^{-1}$ for even $n$. Therefore, the sequence $\left(x_{n}\right)$ diverges unless $\alpha_{2 n} \rightarrow 1$. 
If we consider the explicit method that defines a sequence $\left(x_{n}\right)$ by the iteration process:

$$
x_{n+1}=\alpha_{n} f\left(x_{n}\right)+\left(1-\alpha_{n}\right) x_{n}^{T},
$$

where the initial guess $x_{0} \in \mathbb{R}$ is arbitrary, $\left(\alpha_{n}\right) \subset(0,1)$ and $x_{n}^{T} \in T x_{n}$. Again, if we take $f(x) \equiv u \neq x_{0}$ and $x_{n}^{T}=x_{n}$, then we have $x_{n+1}=$ $\alpha_{n} u+\left(1-\alpha_{n}\right) x_{n}$. It turns out that:

$$
x_{n+1}-u=\left(1-\alpha_{n}\right)\left(x_{n}-u\right)=\cdots=\left(\prod_{i=0}^{n}\left(1-\alpha_{i}\right)\right)\left(x_{0}-u\right) .
$$

Consequently, if we take $\left(\alpha_{n}\right)$,such that $\sum_{n=0}^{\infty} \alpha_{n}=\infty$ (e.g., $\alpha_{n}=\frac{1}{(n+1)^{\alpha}}$ with $0<\alpha \leq 1)$, then the sequence $\left(x_{n}\right)$ diverges.

This simple example shows that iterative methods for a multivalued nonexpansive mapping $T$ may diverge if $T$ is not single valued on its set of fixed points. We will study convergence of implicit and explicit methods for VI (3.2) under the condition that the multivalued nonexpansive mapping is single valued on its set of fixed points.

We begin with the demiclosedness principle for multivalued nonexpansive mappings in a Banach space with Opial's condition.

Lemma 3.2. [3, Theorem 3.1] Let $C$ be a nonempty closed convex subset of a Banach space $X$ which satisfies the Opial condition (2.1). Let $T: C \rightarrow K(C)$ be a multivalued nonexpansive mapping with fixed points. Let $\left(y_{n}\right)_{n \in \mathbb{N}}$ be a bounded sequence, such that:

$$
d\left(y_{n}, T y_{n}\right) \rightarrow 0 \quad \text { as } n \rightarrow \infty .
$$

Then, the weak cluster points of $\left(y_{n}\right)_{n \in \mathbb{N}}$ belong to Fix $(T)$ (i.e., $\omega_{w}\left(y_{n}\right) \subset$ Fix $(T))$.

\subsection{Implicit method}

In this subsection, we consider an implicit method for solving the VI (3.2) in a Banach space satisfying Opial's condition.

Theorem 3.3. Let $X$ be a reflexive Banach space satisfying Opial's condition and having a duality map $J_{\varphi}$ (for some gauge $\varphi$ ) weakly continuous at zero. Let $T: X \rightarrow K(X)$ be a multivalued nonexpansive mappings, such that $F i x(T) \neq \emptyset$ and $T x=\{x\}$ for all $x \in F i x(T)$. Let $D: X \rightarrow X$ be an $\eta$-strongly accretive and $\kappa$-strictly pseudocontractive for some $\eta, \kappa \in(0,1)$, such that $\eta+\kappa>1$. Let $\left(\mu_{n}\right)_{n \in \mathbb{N}} \subset(0,1]$ and let $\left(\alpha_{n}\right)_{n \in \mathbb{N}} \subset(0,1]$ satisfy the condition: $\alpha_{n} \rightarrow 0$ as $n \rightarrow \infty$. Then, the implicit iteration

$$
x_{n}=\alpha_{n}\left(I-\mu_{n} D\right) x_{n}+\left(1-\alpha_{n}\right) x_{n}^{T},
$$

where $x_{n}^{T} \in T x_{n}$, is well defined and strongly converges, as $n \rightarrow \infty$, to the unique solution of the variational inequality:

$$
\left\langle D x^{*}, J\left(x-x^{*}\right)\right\rangle \geq 0 \quad \text { for all } x \in F i x(T) .
$$


Proof. For any $n \in \mathbb{N}$, let us consider the multivalued mappings:

$$
\Gamma_{n}:=\alpha_{n}\left(I-\mu_{n} D\right)+\left(1-\alpha_{n}\right) T .
$$

It is easy to verify that $\Gamma_{n}$ is a contraction. Indeed, setting $w:=\left(I-\mu_{n} D\right) x$ and $v:=\left(I-\mu_{n} D\right) y$ for $x, y \in X$, and applying Lemma 2.1 to $\Gamma_{n} x$ and $\Gamma_{n} y$, we obtain that:

$$
\begin{aligned}
H\left(\Gamma_{n} x, \Gamma_{n} y\right) & \leq \alpha_{n}\|w-v\|+\left(1-\alpha_{n}\right) H(T x, T y) \\
& \leq \alpha_{n}\left\|\left(I-\mu_{n} D\right) x-\left(I-\mu_{n} D\right) y\right\|+\left(1-\alpha_{n}\right) H(T x, T y) \\
& \leq \alpha_{n}\left(1-\mu_{n} \rho\right)\|x-y\|+\left(1-\alpha_{n}\right)\|x-y\| \text { (by Proposition 2.5(ii)) } \\
& =\left(1-\alpha_{n} \mu_{n} \rho\right)\|x-y\| .
\end{aligned}
$$

Then, by Nadler's multivalued contraction fixed point theorem [2], $\Gamma_{n}$ has a fixed point; hence, the implicit scheme (3.1) is well defined. Let $p \in \operatorname{Fix}(T)$. Since $T p=\{p\}$ by assumption, we have:

$$
\left\|x_{n}^{T}-p\right\|=d\left(x_{n}^{T}, T p\right) \leq H\left(T x_{n}, T p\right) \leq\left\|x_{n}-p\right\| .
$$

It follows that:

$$
\begin{aligned}
\left\|x_{n}-p\right\| & =\left\|\alpha_{n}\left(I-\mu_{n} D\right) x_{n}+\left(1-\alpha_{n}\right) x_{n}^{T}-p\right\| \\
& \leq \alpha_{n}\left\|\left(I-\mu_{n} D\right) x_{n}-\left(I-\mu_{n} D\right) p\right\|+\alpha_{n} \mu_{n}\|D p\|+\left(1-\alpha_{n}\right)\left\|x_{n}-p\right\| \\
& \leq \alpha_{n}\left(1-\mu_{n} \rho\right)\left\|x_{n}-p\right\|+\alpha_{n} \mu_{n}\|D p\|+\left(1-\alpha_{n}\right)\left\|x_{n}-p\right\| \\
& =\left(1-\mu_{n} \alpha_{n} \rho\right)\left\|x_{n}-p\right\|+\alpha_{n} \mu_{n}\|D p\| .
\end{aligned}
$$

It turns out that:

$$
\left\|x_{n}-p\right\| \leq \frac{\|D p\|}{\rho} .
$$

In particular, the sequence $\left(x_{n}\right)$ is bounded. Moreover, for $w \in F i x(T)$, we have:

$$
\begin{aligned}
\left\|x_{n}-w\right\|^{2} & =\left\langle x_{n}-w, J\left(x_{n}-w\right)\right\rangle \\
& =\alpha_{n}\left\langle x_{n}-\mu_{n} D x_{n}-w, J\left(x_{n}-w\right)\right\rangle+\left(1-\alpha_{n}\right)\left\langle x_{n}^{T}-w, J\left(x_{n}-w\right)\right\rangle \\
& \leq \alpha_{n}\left\langle x_{n}-w-\mu_{n} D x_{n}, J\left(x_{n}-w\right)\right\rangle+\left(1-\alpha_{n}\right)\left\|x_{n}^{T}-w\right\| \cdot\left\|x_{n}-w\right\| \\
& \leq\left\|x_{n}-w\right\|^{2}-\alpha_{n} \mu_{n}\left\langle D x_{n}, J\left(x_{n}-w\right)\right\rangle .
\end{aligned}
$$

It turns out that:

$$
\left\langle D x_{n}, J\left(x_{n}-w\right)\right\rangle \leq 0, \quad w \in F i x(T) .
$$

Now, since $D$ is $\eta$-strongly accretive, it follows from (3.5) that:

$$
\begin{aligned}
0 & \geq\left\langle D x_{n}-D w, J\left(x_{n}-w\right)\right\rangle+\left\langle D w, J\left(x_{n}-w\right)\right\rangle \\
& \geq \eta\left\|x_{n}-w\right\|^{2}+\left\langle D w, J\left(x_{n}-w\right)\right\rangle .
\end{aligned}
$$

This implies that:

$$
\left\|x_{n}-w\right\|^{2} \leq-\frac{1}{\eta}\left\langle D w, J\left(x_{n}-w\right)\right\rangle .
$$

Since $\left(x_{n}\right)$ is bounded and $\alpha_{n} \rightarrow 0$ and using (3.3) and (3.1), we see that $\left(x_{n}^{T}\right)$ is bounded, and

$$
\left\|x_{n}-x_{n}^{T}\right\|=\alpha_{n}\left\|\left(I-\mu_{n} D\right) x_{n}-x_{n}^{T}\right\| \rightarrow 0 \text { as } n \rightarrow \infty .
$$


Consequently, $d\left(x_{n}, T x_{n}\right) \rightarrow 0$ as $n \rightarrow \infty$. By Lemma 3.2, every weak limit point $z$ of $\left(x_{n}\right)_{n \in \mathbb{N}}$ is a fixed point of $T$. We claim that $z$ also solves VI (3.2). To verify this, take a subsequence $\left(x_{n_{k}}\right)$ of $\left(x_{n}\right)$, such that $x_{n_{k}} \rightarrow z$, which implies that $J_{\varphi}\left(x_{n_{k}}-z\right) \stackrel{*}{\rightarrow} 0$ by the assumption that $J_{\varphi}$ is weakly continuous at zero. Using (2.2), we can equivalently rewrite (3.5) and (3.6) as:

$$
\left\langle D x_{n}, J_{\varphi}\left(x_{n}-w\right)\right\rangle \leq 0, \quad w \in F i x(T)
$$

and respectively:

$$
\varphi\left(\left\|x_{n}-w\right\|\right) \leq-\frac{1}{\eta}\left\langle D w, J_{\varphi}\left(x_{n}-w\right)\right\rangle, \quad w \in F i x(T) .
$$

Passing to the subsequence $\left(x_{n_{k}}\right)$, we get:

$$
\left\langle D x_{n_{k}}, J_{\varphi}\left(x_{n_{k}}-w\right)\right\rangle \leq 0, \quad w \in F i x(T)
$$

and

$$
\varphi\left(\left\|x_{n_{k}}-z\right\|\right) \leq-\frac{1}{\eta}\left\langle D w, J_{\varphi}\left(x_{n_{k}}-z\right)\right\rangle .
$$

We then obtain $x_{n_{k}} \rightarrow z$ in norm from (3.10) and furthermore from (3.9):

$$
\left\langle D z, J_{\varphi}(z-w)\right\rangle \leq 0, \quad w \in F i x(T) .
$$

This proves that $z$ solves VI (3.2); hence, $z=x^{*}$ by the uniqueness of solutions of VI (3.2). Therefore, we have proved that $x_{n} \rightarrow x^{*}$ in norm.

Corollary 3.4. Suppose $X$ is a reflexive Banach space. Suppose, in addition, $X$ either satisfies Opial's condition and has a uniformly Gateaux differentiable norm or has a weakly continuous duality map $J_{\varphi}$ for some gauge $\varphi$. Then, the conclusion of Theorem 3.3 holds.

Proof. In either case, we see that $X$ satisfies the assumptions in Theorem 3.3 , i.e., Opial's condition and a duality map $J_{\varphi}$ weakly continuous at zero. Indeed, this follows from Lemma 2.3 in the first case and trivially in the second case.

\subsection{Explicit method}

To define an explicit algorithm, let us recall a relevant property regarding the Hausdorff metric.

Lemma 3.5. [2, p. 480] Let $(M, d)$ be a complete metric space and $A, B$ compact subset of $M$. Then, for any $a \in A$, there exists $b \in B$, such that:

$$
d(a, b) \leq H(A, B) .
$$

Now, consider VI (3.2) where we assume $T: X \rightarrow K(X)$ has a nonempty set of fixed points. Take an arbitrary starting point $x_{0} \in X$ to define explicitly a sequence $\left(x_{n}\right)$ by the procedure:

$$
x_{n+1}=\lambda_{n} x_{n}+\left(1-\lambda_{n}\right) x_{n}^{T}-\left(1-\lambda_{n}\right) \mu_{n} D x_{n}^{T},
$$

where $x_{n}^{T} \in T x_{n}$ is chosen in such a way (guaranteed by Lemma 3.5) that:

$$
\left\|x_{n}^{T}-x_{n-1}^{T}\right\| \leq H\left(T x_{n}, T x_{n-1}\right)
$$

for $n \geq 1$.

The next Lemma, proved in [40], will be used in our proof. 
Lemma 3.6. Assume $\left(b_{n}\right)_{n \in \mathbb{N}}$ is a sequence of nonnegative numbers for which:

$$
b_{n+1} \leq\left(1-a_{n}\right) b_{n}+\delta_{n}, \quad n \geq 0,
$$

where $\left(a_{n}\right)_{n \in \mathbb{N}}$ is a sequence in $(0,1)$ and $\left(\delta_{n}\right)_{n \in \mathbb{N}}$ is a sequence in $\mathbb{R}$, such that:

1. $\sum_{n=1}^{\infty} a_{n}=\infty$;

2. $\limsup _{n \rightarrow \infty} \frac{\delta_{n}}{a_{n}} \leq 0$ or $\sum_{n=1}^{\infty}\left|\delta_{n}\right|<\infty$.

Then, $\lim _{n \rightarrow \infty} b_{n}=0$.

Theorem 3.7. Let $X$ be a reflexive space with a weakly sequentially continuous duality mapping $J_{\varphi}$. Let $T: X \rightarrow K(X)$ a nonexpansive multivalued mapping, such that $F i x(T)$ is nonempty and $T x=\{x\}$ for all $x \in F i x(T)$. Let $D$ : $X \rightarrow X$ an $\eta$-strongly accretive and $\kappa$-strictly pseudocontractive, such that $\eta+\kappa>1$. Let $\left(\mu_{n}\right)_{n \in \mathbb{N}} \subset(0,1]$ and $\left(\lambda_{n}\right)_{n \in \mathbb{N}} \subset[0, a]$ for some $a \in(0,1)$, such that:

(i) $\lim _{n \rightarrow \infty} \mu_{n}=0$ and $\sum_{n=0}^{\infty} \mu_{n}=\infty$.

(ii) $\lim _{n \rightarrow \infty} \frac{\left|\lambda_{n}-\lambda_{n-1}\right|}{\mu_{n}}=0$.

(iii) $\lim _{n \rightarrow \infty} \frac{\left|\mu_{n}-\mu_{n-1}\right|}{\mu_{n}}=0$.

Then, the sequence $\left(x_{n}\right)$ defined by the explicit iteration (3.12) strongly converges, as $n \rightarrow \infty$, to the unique solution of the VI (3.2).

Proof. At first, defining $B_{n}:=\left(I-\mu_{n} D\right)$ rewrites our iteration (3.12) as:

$$
x_{n+1}=\lambda_{n} x_{n}+\left(1-\lambda_{n}\right) B_{n} x_{n}^{T} .
$$

Notice that each $B_{n}$ is a contraction by Proposition 2.5 (ii).

Take $p \in F i x(T)$ to derive that (noting $T p=\{p\}$ by assumption which implies that $\left.\left\|x_{n}^{T}-p\right\| \leq\left\|x_{n}-p\right\|\right)$ :

$$
\begin{aligned}
\left\|x_{n+1}-p\right\| & \leq \lambda_{n}\left\|x_{n}-p\right\|+\left(1-\lambda_{n}\right)\left\|B_{n} x_{n}^{T}-B_{n} p\right\|+\left(1-\lambda_{n}\right) \mu_{n}\|D p\| \\
& \leq \lambda_{n}\left\|x_{n}-p\right\|+\left(1-\lambda_{n}\right)\left(1-\mu_{n} \rho\right)\left\|x_{n}-p\right\|+\left(1-\lambda_{n}\right) \mu_{n}\|D p\| \\
& =\left(1-\left(1-\lambda_{n}\right) \mu_{n} \rho\right)\left\|x_{n}-p\right\|+\left(1-\lambda_{n}\right) \mu_{n} \rho(\|D p\| / \rho) \\
& \leq \max \left\{\left\|x_{n}-p\right\|,\|D p\| / \rho\right\} \\
& \leq \ldots \leq \max \left\{\left\|x_{1}-p\right\|,\|D p\| / \rho\right\} .
\end{aligned}
$$

It turns out that $\left(x_{n}\right)$ is bounded. 
The next step is to claim that $\omega_{w}\left(x_{n}\right) \subset F i x(T)$, for which we use (3.14) to estimate $\left\|x_{n+1}-x_{n}\right\|$ as follows:

$$
\begin{aligned}
\left\|x_{n+1}-x_{n}\right\|= & \left\|\lambda_{n} x_{n}+\left(1-\lambda_{n}\right) B_{n} x_{n}^{T}-\left(\lambda_{n-1} x_{n-1}+\left(1-\lambda_{n-1}\right) B_{n-1} x_{n-1}^{T}\right)\right\| \\
= & \| \lambda_{n}\left(x_{n}-x_{n-1}\right)+\left(1-\lambda_{n}\right)\left(B_{n} x_{n}^{T}-B_{n-1} x_{n-1}\right) \\
& +\left(\lambda_{n}-\lambda_{n-1}\right)\left(x_{n-1}-B_{n-1} x_{n-1}^{T}\right) \| \\
\leq & \lambda_{n}\left\|x_{n}-x_{n-1}\right\|+\left|\lambda_{n}-\lambda_{n-1}\right|\left\|x_{n-1}-B_{n-1} x_{n-1}^{T}\right\| \\
& +\left(1-\lambda_{n}\right)\left\|B_{n} x_{n}^{T}-B_{n-1} x_{n-1}^{T}\right\| \\
\leq & \lambda_{n}\left\|x_{n}-x_{n-1}\right\|+\left|\lambda_{n}-\lambda_{n-1}\right|\left\|x_{n-1}-B_{n-1} x_{n-1}^{T}\right\| \\
& +\left(1-\lambda_{n}\right)\left(\left\|B_{n} x_{n}^{T}-B_{n} x_{n-1}^{T}\right\|+\left\|B_{n} x_{n-1}^{T}-B_{n-1} x_{n-1}^{T}\right\|\right) .
\end{aligned}
$$

Now, since $\left(x_{n}\right)$ is bounded, we have a constant $M>0$, such that:

$$
\max \left\{\left\|x_{n}-B_{n} x_{n}^{T}\right\|,\left\|D x_{n}^{T}\right\|: n \geq 0\right\} \leq M .
$$

It follows from Proposition 2.5(ii):

$$
\begin{aligned}
\left\|B_{n} x_{n}^{T}-B_{n} x_{n-1}^{T}\right\| & \leq\left(1-\mu_{n} \rho\right)\left\|x_{n}^{T}-x_{n-1}^{T}\right\| \leq \rho\left\|x_{n}-x_{n-1}\right\| \\
\left\|B_{n} x_{n-1}^{T}-B_{n-1} x_{n-1}^{T}\right\| & =\left|\mu_{n}-\mu_{n-1}\right|\left\|D x_{n-1}^{T}\right\| \leq M\left|\mu_{n}-\mu_{n-1}\right| .
\end{aligned}
$$

Substituting (3.16-3.17) into (3.15) yields:

$\left.\left\|x_{n+1}-x_{n}\right\| \leq\left[1-\left(1-\lambda_{n}\right) \mu_{n} \rho\right)\right]\left\|x_{n}-x_{n-1}\right\|+M\left(\left|\lambda_{n}-\lambda_{n-1}\right|+\left|\mu_{n}-\mu_{n-1}\right|\right)$.

Setting

$b_{n}=\left\|x_{n}-x_{n-1}\right\|, \quad a_{n}=\rho \mu_{n}\left(1-\lambda_{n}\right), \quad \delta_{n}=M\left(\left|\lambda_{n}-\lambda_{n-1}\right|+\left|\mu_{n}-\mu_{n-1}\right|\right)$,

we can rewrite the last relation as:

$$
b_{n+1} \leq\left(1-a_{n}\right) b_{n}+\delta_{n} .
$$

It is easily seen from conditions (i)-(iii) that $\left(a_{n}\right)$ and $\left(\delta_{n}\right)$ satisfy the conditions $\sum_{n=0}^{\infty} a_{n}=\infty$ and $\lim _{n \rightarrow \infty} \delta_{n} / a_{n}=0$. Consequently, we can apply Lemma 3.6 to obtain that $\lim _{n \rightarrow \infty} b_{n}=0$; namely, $\lim _{n \rightarrow \infty}\left\|x_{n+1}-x_{n}\right\|=0$.

Now, we have:

$$
\begin{aligned}
\left\|x_{n}-x_{n}^{T}\right\| & \leq\left\|x_{n}-x_{n+1}\right\|+\left\|x_{n+1}-x_{n}^{T}\right\| \\
& \leq\left\|x_{n}-x_{n+1}\right\|+\left\|\lambda_{n}\left(x_{n}-x_{n}^{T}\right)+\left(1-\lambda_{n}\right) \mu_{n} D x_{n}^{T}\right\| \\
& \leq\left\|x_{n}-x_{n+1}\right\|+\lambda_{n}\left\|x_{n}-x_{n}^{T}\right\|+\left(1-\lambda_{n}\right) \mu_{n}\left\|D x_{n}^{T}\right\| .
\end{aligned}
$$

It follows that:

$$
\left\|x_{n}-x_{n}^{T}\right\| \leq \frac{1}{1-\lambda_{n}}\left\|x_{n}-x_{n+1}\right\|+\mu_{n}\left\|D x_{n}^{T}\right\| \rightarrow 0,
$$

since $\mu_{n} \rightarrow 0$ and $0 \leq \lambda_{n} \leq a$ for some $0<a<1$. This implies that:

$$
d\left(x_{n}, T x_{n}\right) \leq\left\|x_{n}-x_{n}^{T}\right\| \rightarrow 0 .
$$


Consequently, Lemma 3.2 ensures that every weak limit of $\left(x_{n}\right)$ is a fixed point of $T$. To prove the strong convergence of $\left(x_{n}\right)$, let $x^{*} \in$ Fix $(T)$ denote the unique solution of VI (3.2). Since $J_{\varphi}$ is the subdifferential of $\Phi$, we have:

$$
\begin{aligned}
\Phi\left(\left\|x_{n+1}-x^{*}\right\|\right)= & \Phi\left(\left\|\lambda_{n}\left(x_{n}-x^{*}\right)+\left(1-\lambda_{n}\right)\left(B_{n} x_{n}^{T}-x^{*}\right)\right\|\right) \\
= & \left.\Phi\left(\| \lambda_{n}\left(x_{n}-x^{*}\right)+\left(1-\lambda_{n}\right)\left(B_{n} x_{n}^{T}-B_{n} x^{*}\right)-\left(1-\lambda_{n}\right) \mu_{n} D x^{*}\right) \|\right) \\
\leq & \Phi\left(\left\|\lambda_{n}\left(x_{n}-x^{*}\right)+\left(1-\lambda_{n}\right)\left(B_{n} x_{n}^{T}-B_{n} x^{*}\right)\right\|\right) \\
& -\left(1-\lambda_{n}\right) \mu_{n}\left\langle D x^{*}, J_{\varphi}\left(x_{n+1}-x^{*}\right)\right\rangle \\
\leq & \lambda_{n} \Phi\left(\left\|x_{n}-x^{*}\right\|\right)+\left(1-\lambda_{n}\right)\left(1-\mu_{n} \rho\right) \Phi\left(\left\|x_{n}^{T}-x^{*}\right\|\right) \\
& -\left(1-\lambda_{n}\right) \mu_{n}\left\langle D x^{*}, J_{\varphi}\left(x_{n+1}-x^{*}\right)\right\rangle \\
\leq & {\left[1-\left(1-\lambda_{n}\right) \mu_{n} \rho\right] \Phi\left(\left\|x_{n}-x^{*}\right\|\right) } \\
& -\left(1-\lambda_{n}\right) \mu_{n}\left\langle D x^{*}, J_{\varphi}\left(x_{n+1}-x^{*}\right)\right\rangle \\
= & \left(1-a_{n}\right) \Phi\left(\left\|x_{n}-x^{*}\right\|\right)+a_{n} \gamma_{n},
\end{aligned}
$$

where

$$
a_{n}=\rho \mu_{n}\left(1-\lambda_{n}\right), \quad \gamma_{n}=\frac{\left\langle-D x^{*}, J_{\varphi}\left(x_{n+1}-x^{*}\right)\right\rangle}{\rho} .
$$

Now, take a subsequence $\left(x_{n_{k}}\right)$ of $\left(x_{n}\right)$, such that:

$$
\limsup _{n \rightarrow \infty}\left\langle-D w, J_{\varphi}\left(x_{n}-x^{*}\right)\right\rangle=\lim _{k \rightarrow \infty}\left\langle-D x^{*}, J_{\varphi}\left(x_{n_{k}}-x^{*}\right)\right\rangle .
$$

We may further assume $x_{n_{k}} \rightarrow \hat{x}$ weakly. As a result, the last relation is reduced to:

$$
\limsup _{n \rightarrow \infty}\left\langle-D w, J_{\varphi}\left(x_{n}-x^{*}\right)\right\rangle=\left\langle-D x^{*}, J_{\varphi}\left(\hat{x}-x^{*}\right)\right\rangle \leq 0,
$$

since $x^{*}$ is the solution of the VI (3.2) and $\hat{x} \in F i x(T)$. In other words, we have $\lim \sup _{n \rightarrow \infty} \gamma_{n} \leq 0$. Consequently, Lemma 3.6 is applicable to (3.19) to get $\Phi\left(\left\|x_{n}-x^{*}\right\|\right) \rightarrow 0$, or $x_{n} \rightarrow x^{*}$ in norm. This finishes the proof.

Remark 3.8. The choices of $\left(\mu_{n}\right)$ and $\left(\lambda_{n}\right)$ given by $\mu_{n}=(n+1)^{-\mu}$ and $\lambda_{n}=(n+1)^{-\lambda}$ with $0<\mu \leq 1$ and $\lambda+1-\mu>0$ satisfy the conditions (i)-(iii) in Theorem 3.7 .

\subsection{Open questions}

We conclude the paper by raising some open problems which we think are of interest.

(i) Does the conclusion of Theorem 3.3 hold under weaker conditions on the underlying Banach space $X$ ? In particular, $X$ is assumed to satisfy Opial's condition only.

(ii) Does the conclusion of Theorem 3.7 hold under conditions weaker than the condition of a duality map $J_{\varphi}$ being weakly continuous? In particular, $X$ is assumed to satisfy Opial's condition only.

\section{Acknowledgements}

This work was completed, while the second author were visiting the School of Science, Hangzhou Dianzi University, Hangzhou, China. The second author is grateful for the hospitality. The first author was supported in part by an Australian Research Council/Discovery Project DP 200100124. 
Funding Open access funding provided by Università della Calabria within the CRUI-CARE Agreement.

Open Access. This article is licensed under a Creative Commons Attribution 4.0 International License, which permits use, sharing, adaptation, distribution and reproduction in any medium or format, as long as you give appropriate credit to the original author(s) and the source, provide a link to the Creative Commons licence, and indicate if changes were made. The images or other third party material in this article are included in the article's Creative Commons licence, unless indicated otherwise in a credit line to the material. If material is not included in the article's Creative Commons licence and your intended use is not permitted by statutory regulation or exceeds the permitted use, you will need to obtain permission directly from the copyright holder. To view a copy of this licence, visit http:// creativecommons.org/licenses/by/4.0/.

Publisher's Note Springer Nature remains neutral with regard to jurisdictional claims in published maps and institutional affiliations.

\section{References}

[1] Goebel, K., Reich, S.: Uniform convexity, hyperbolic geometry, and nonexpansive mappings. Dekker, New York (1984)

[2] Nadler, S.: Multi-valued contraction mappings. Pac. J. Math. 30(2), 475-488 (1969)

[3] Lami, Dozo E.: Multivalued nonexpansive mappings and Opial condition. Proc. Am. Math. Soc. 38(2), 286-292 (1973)

[4] Lim, T.C.: A fixed point theorem for multivalued nonexpansive mappings in a uniformly convex Banach space. Bull. Am. Math. Soc. 80(6), 1123-1126 (1974)

[5] Ko, H.M.: Fixed point theorems for point-to-set mappings and the set of fixed points. Pac. J. Math. 42(2), 369-379 (1972)

[6] Xu, H.K.: Metric fixed point theory for multivalued mappings. Dissert. Math. 389, 39 (2000)

[7] Reich, S.: Approximate selections, best approximations, fixed points, and invariant sets. J. Math. Anal. Appl. 62, 104-113 (1978)

[8] Pietramala, P.: Convergence of approximating fixed points sets for multivalued nonexpansive mappings. Comment. Math. Univ. Carolin 32(4), 697-701 (1991)

[9] Browder, F.E.: Convergence of approximants to fixed points of nonexpansive nonlinear mappings in Banach spaces. Arch. Rat. Mech. and Anal. 24(1), 82-90 (1967)

[10] López, Acedo G., Xu, H.K.: Remarks on multivalued nonexpansive mappings. Soochow J. Mathe. 21(1), 107-115 (1995)

[11] Sahu, D.R.: Strong convergence theorems for nonexpansive type and non-self multi-valued mappings. Nonlinear Anal. TMA 37(3), 401-407 (1999)

[12] Jung, J.S.: Strong convergence theorems for multivalued nonexpansive nonselfmappings in Banach spaces. Nonlinear Anal. TMA 66(11), 2345-2354 (2007) 
[13] Shahzad, N., Zegeye, H.: Strong convergence results for nonself multimaps in Banach spaces. Proc. Am. Math. Soc. 136(2), 539-548 (2007)

[14] Shahzad, N., Zegeye, H.: On Mann and Ishikawa iteration schemes for multivalued maps in Banach spaces. Nonlinear Anal. TMA 71(3-4), 838-844 (2009)

[15] Zegeye, H., Shahzad, N.: Viscosity approximation methods for nonexpansive multimaps in Banach spaces. Acta Math. Sin, English Ser. 26(6), 1165-1176 (2010)

[16] Reich, S.: Strong convergence theorems for resolvents of accretive operators in Banach spaces. J. Math. Anal. Appl. 75(1), 287-292 (1980)

[17] Reich, S.: Extension problems for accretive sets in Banach spaces. J. Funct. Anal. 26, 378-395 (1977)

[18] Kopecká, E., Reich, S.: Nonexpansive retracts in Banach spaces. Banach Center Publ. 77, 161-174 (2007)

[19] Attouch, H.: Viscosity approximation methods for minimization problems. SIAM J. Optim. 6(3), 769-806 (1996)

[20] Moudafi, A.: Viscosity approximation methods for fixed-points problems. J. Math. Anal. Appl. 241(1), 46-55 (2000)

[21] Xu, H.K.: Viscosity approximation methods for nonexpansive mappings. J. Math. Anal. Appl. 298(1), 279-291 (2004)

[22] Cui, Y., Fei, Z.Z., Hudzik, H.: Viscosity approximation methods for multivalued mappings in Banach spaces. Numer. Funct. Anal. Opt. 33(11), 1288-1303 (2012)

[23] Wu, X., Zhao, L.: Viscosity approximation methods for multivalued nonexpansive mappings. Mediterranean J. Math. 13(5), 2645-2657 (2016)

[24] Panyanak B., Suantai S.: Viscosity approximation methods for multivalued nonexpansive mappings in geodesic spaces, Fixed Point Theory and Appl., (1), $114(2015)$

[25] Xu, H.K., Alghamdi, M.A., Shahzad, N.: Remarks on iterative methods for multivalued nonexpansive mappings. J. Nonlinear Conv. Anal. 18(1), 161-171 (2017)

[26] He, H., Liu, S., Chen, R.: Convergence results of multi-valued nonexpansive mappings in Banach spaces, J. Inequal. Appl., (1), 483 (2014)

[27] Takahashi, W.: Nonlinear functional analysis: fixed point theory and its applications. Yokohama publishers, Yokohama (2000)

[28] Opial, Z.: Weak convergence of the sequence of successive approximations for nonexpansive mappings. Bull. Am. Math. Soc. 73(4), 591-597 (1967)

[29] Emmanuele, G.: Asymptotic behavior of iterates of nonexpansive mappings in Banach spaces with Opial's condition. Proc. Am. Math. Soc. 94(1), 103-109 (1985)

[30] Engl, H.W.: Some random fixed point theorems for strict contractions and nonexpansive mappings. Nonlinear Anal. TMA 2(5), 619-626 (1978)

[31] Gossez, J.P., Lami, Dozo E.: Some geometric properties related to the fixed point theory for nonexpansive mappings. Pac. J. Math. 40(3), 565-573 (1972)

[32] Browder, F.E.: Fixed point theorems for nonlinear semicontractive mappings in Banach spaces. Arch. Rat. Mech. Anal. 21(4), 259-269 (1966)

[33] Cioranescu, I.: Geometry of Banach spaces, duality mappings and nonlinear problems, vol. 62. Springer Science \& Business Media, Berlin (2012) 
[34] Xu, H.K., Kim, T.H., Yin, X.: Weak continuity of the normalized duality map. J. Nonlinear Convex Anal. 15(3), 595-604 (2014)

[35] Lim, T.C., Xu, H.K.: Fixed point theorems of asymptotically nonexpansive mappings. Nonlinear Anal. TMA 22(11), 1345-1355 (1994)

[36] Xu, H.K.: Banach space properties of Opial's type and fixed point theorems of nonlinear mappings, Annales Universitatis Mariae Curie-Sklodowska. Sect. A 51(2), 293-303 (1997)

[37] Ceng, L.C., Ansari, Q.H., Yao, J.C.: Mann-type steepest-descent and modified hybrid steepest-descent methods for variational inequalities in Banach spaces. Numer. Funct. Anal. Optim. 29(9-10), 987-1033 (2008)

[38] Xu, H.K., Kim, T.H.: Convergence of hybrid steepest-descent methods for variational inequalities. J. Opt. Theory Appl. 119(1), 185-201 (2003)

[39] Marino, G., Muglia, L., Yao, Y.: The uniform asymptotical regularity of families of mappings and solutions of variational inequality problems. J. Nonlinear Convex Anal. 15(3), 477-492 (2014)

[40] Xu, H.K.: Iterative algorithms for nonlinear operators. J. Lond. Math. Soc. 66(1), 240-256 (2002)

Hong-Kun Xu

School of Science

Hangzhou Dianzi University

Hangzhou 310018

China

e-mail: xuhk@hdu.edu.cn

Luigi Muglia

Dipartimento di Matematica

Universitá della Calabria

87036 Arcavacata di Rende CS

Italy

e-mail: muglia@mat.unical.it 